\title{
Moulded calculus of common bile duct mimicking
} a stenosis

\author{
Andrzej Jamry ${ }^{1}$, Marian Brocki², Jacek Śmigielski \\ ${ }^{1} 2^{\text {nd }}$ Surgical Department, District Hospital, Starachowice, Poland \\ 2Department of Chest, General and Oncological Surgery, University Hospital WAM and CSW, Medical University of Lodz, Poland
}

Prz Gastroenetrol 2014; 9 (2): 116-120

DOI: $10.5114 / p g .2014 .42508$

Key words: choledocholithiasis, endoscopic retrograde cholangiopancreatography, diagnosis differential.

Address for correspondence: Andrzej Jamry MD, PhD, $2^{\text {nd }}$ Surgical Department, District Hospital, 70 Radomska St, 27-200 Starachowice,

Poland, phone: +48 602795 259, e-mail: jamry@tlen.pl

\begin{abstract}
Bile duct stenosis, in most cases, appears to be the consequence of pancreatic head, ampulla of Vater and bile duct tumours, cholangitis sclerosans, as well as iatrogenic damages, which may all be diagnosed during endoscopic retrograde cholangiopancreatography (ERCP). In very rare cases the restriction may result from an atypically shaped wedged stone. This situation creates many diagnostic problems, which in the majority of cases can be solved using imaging studies. However, in some patients even a significant extension of diagnostic procedures may not lead to a correct diagnosis. We present a diagnostically difficult case of a deposit imitating restriction. We present a 70-year-old woman with common bile duct restriction undiagnosed despite several ultrasound examinations (USG), computed tomography (CT), double magnetic resonance cholangiopancreatography (MRCP) and endoscopic retrograde cholangiopancreatography (ERCP). Only after the third ERCP examination a fragmented, by formerly introduced prosthesis, deposit, imitating narrowing, was revealed. Identification of bile duct deposits depends on their composition, localisation and the imaging techniques used. Pigment calculi with atypical shape, bile density, air density or surrounding tissue density are very difficult to diagnose. Thus, the sensitivity of common bile duct stone detection in USG, CT, MRCP and endoscopic ultrasound (EUS) is 5-88\%; 6-88\%; 73-97\%; and 84-98\%, respectively. Moreover, ERCP may not diagnose the character of the restriction even in $5.2 \%$ up to $30 \%$ of the patients. Consequently, assessment of diagnosis in a number of patients is difficult. A deposit imitating common bile duct (CBD) restriction is a rare, difficult to diagnose phenomenon, which should be taken into account during differential diagnosis of CBD restrictions.
\end{abstract}

\section{Introduction}

The most common causes of bile duct stenosis observed in endoscopic retrograde cholangiopancreatography (ERCP) are pancreatic head, ampulla of Vater and bile duct tumours, chronic pancreatitis, hepatic metastatic tumours, cholangitis sclerosans and iatrogenic damage. Rarely, the restriction may be the reason for infection, histiocytosis, Alagille syndrome, transplant against host reaction, chemotherapy in the past and hepatic hilum or common bile duct (CBD) cysts. Very rarely, the stenosis may arise from an atypical shape of the wedged deposit, without a meniscus in the cholangiography. Correct diagnosis in such cases is problematic. It seems that other imaging techniques may be useful; however, the density of the deposits may be identical to tissue, air or bile masses, which is why they are not visible in available imaging examinations [1-4].
We present diagnostic difficulties of a moulding deposit in the proximal part of the CBD, imitating a stenosis.

\section{Case report}

A 70-year-old woman was referred to the hospital due to the obstructive jaundice and serious widening of the CBD with the loss of filling, corresponding to a deposit seen in ultrasound examinations (USG). The patient had undergone cholecystectomy 32 years before and endoscopic sphincterotomy and removal of deposits several years later. Endoscopic retrograde cholangiopancreatography revealed ampulla of Vater restenosis and proximal narrowing in the form of obverse meniscus and constricted passage proximal to the widened intrahepatic ducts. The two balloting deposits in the distal part were removed (Figure 1). The sphinc- 


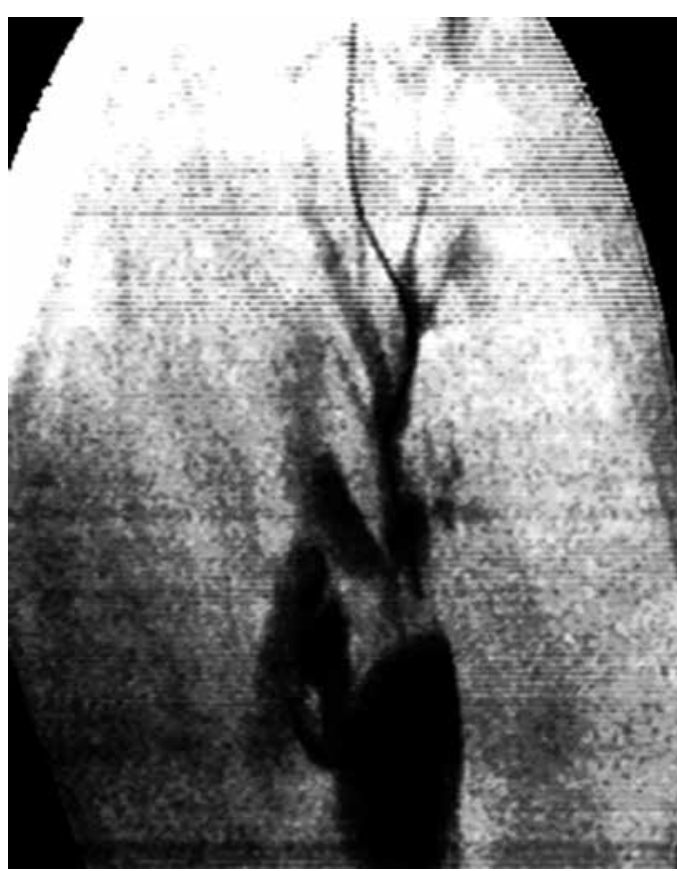

Figure 1. Moulded calculus mimicking a stenosis

terotomy was expanded and a prosthesis introduced. Computed tomography (CT) presented no pathology in CBD, which was $10 \mathrm{~mm}$ in diameter. Therefore, the prosthesis was explanted with a gastroscope, and no bile duct control was performed (the procedure was performed in a different centre). Proper ultrasonography results were achieved 2 months later, but magnetic resonance cholangiopancreatography (MRCP), executed 1 day later, presented a CBD $13 \mathrm{~mm}$ in diameter with various dimensions of low density areas, suggestive of deposits. A second ERCP procedure was performed to resolve diverse results, which presented only the air in the proximal part of the unchanged, restricted CBD. A prosthesis was implanted again. The next MRCP procedure was carried out 1 month later, and only air was found in the bile ducts. Having divergent results, the patient was qualified to transoral cholangioscopy. Cholangiography was conducted as a prelude to cholangioscopy, and the stenosis was not found; instead, many fragile, fragmented deposits and air bubbles were visible during the trials of concrement removal (Figure 2). The examination demonstrated that the narrowing was the effect of low density, moulding pigment deposits, fragmented by the prosthesis in the bile duct tree (Figure 3).

\section{Discussion}

The efficacy of imaging techniques in bile duct deposit detection depends on their composition, size, shape and localisation, or the presence of concomitant stenosis. The concrements found in bile ducts may mi-

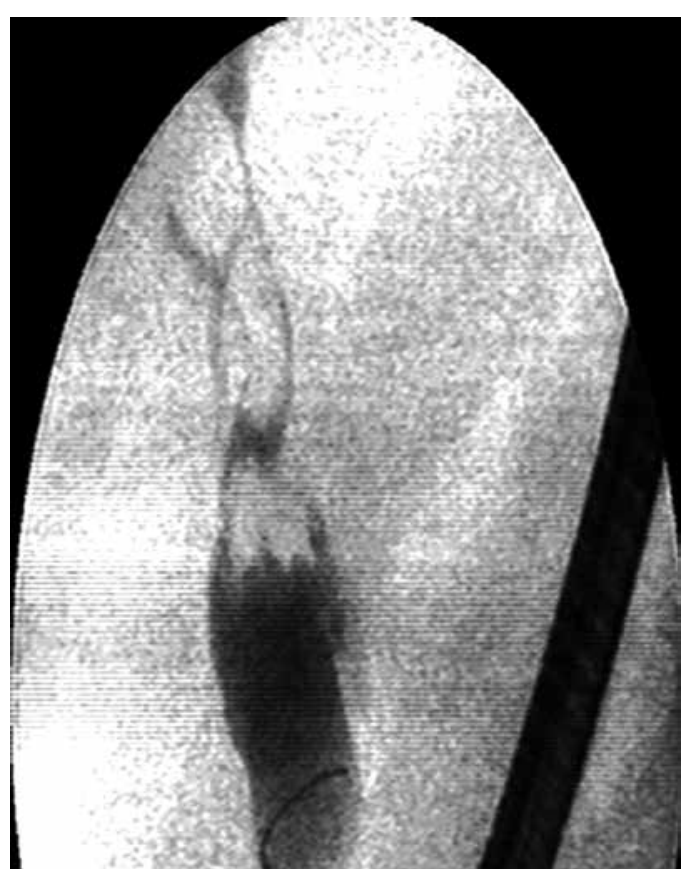

Figure 2. Fragmented calculus

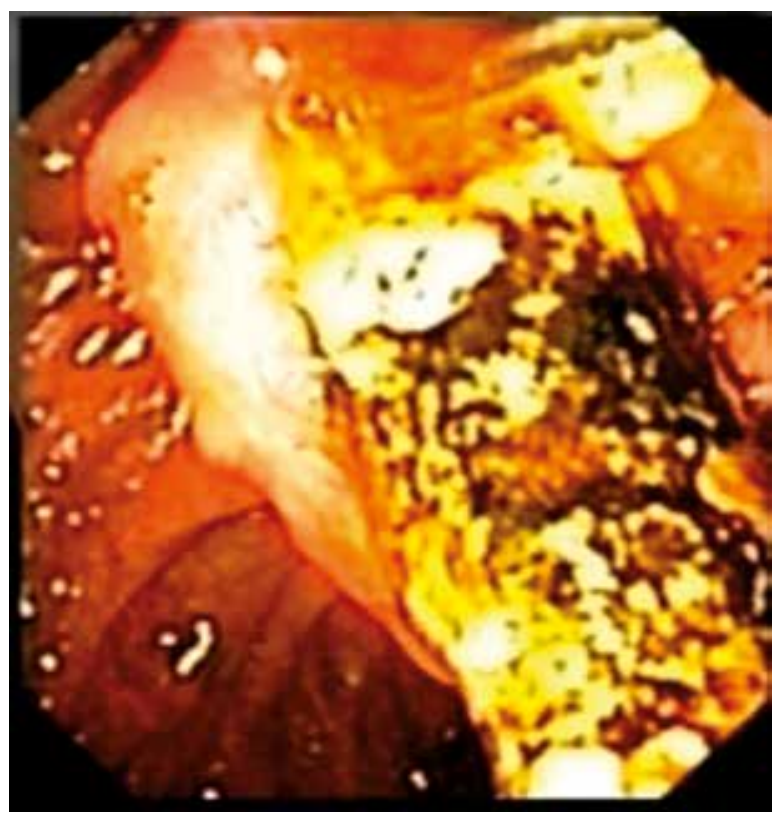

Figure 3. Removed calculus

grate from bile vesicle (31\%), or grow primarily in bile duct lumen (brown bilirubine $54.3 \%$, black bilirubine $11.8 \%$, and mixed 2.8\%) [2]. Most of the concrements occurring in the vesicle are formed from cholesterol (70-90\% of cases) by non-bacterial haemolysis of bilirubine bindings. The deposits formed primarily in the bile ducts are most often soft, pigment and brown, and they contain 10-60\% calcium bilirubinate and less 
than $20 \%$ cholesterol. These features promote various shapes, and imitations of CBD stenosis in 1 out of 33 cases. Their formation is connected with the amplified number of bacteria producing hydrolytic enzymes, such as $\beta$-glucuronidase, phospholipase $A$ and hydrolase. The process occurs most often in patients with deteriorated bile drainage in the course of Oddie sphincter dysfunction, primary sclerotic cholangitis (PSC) and iatrogenic damage, ampulla of Vater localised in the diverticulum or sphincterectomy in the past $[3,5]$. The third type of deposit presents a mixed texture with similar content of cholesterol and calcium bilirubinate. It is hard, round or elliptic with a smooth, black surface. The reason for the lithiasis recurrence in the presented case might have been ampulla of Vater restenosis leading to infection and the formation of pigment deposits imitating stenosis.

Bile deposits present an echogenic line, responding to the anterior edge of such a deposit, with the distal shade or the whole deposit with non-homogenous echogenicity in ultrasound. The deposits without acoustic shade account for $25 \%$ of all concrements, and the phenomenon occurs in bile pigment deposits, irrespective of their dimensions $[3,6]$. This is caused by the similar density of the concrements and surrounding bile. Additionally, ultrasound sensitivity of stone detection is decreased in the proximal CBD, due to imaging difficulties in the proximity of the duodenum [3]. Moreover, the image interpretation may be impeded by the presence of air bubbles, mucus conglomerates, blood thrombi, post procedural clips, parasites, inflammatory infiltration or anatomical anomalies $[3,6]$. The results are also dependent on CBD diameter, obesity of the patient, amount of intestinal gases and the operator's experience $[1,6,7]$. Therefore, sensitivity of ultrasound in CBD deposit detection ranges from $5 \%$ to $88 \%$, and specificity from $68 \%$ to $91 \%[1,3,6-8]$. The reported difficulties also influence the possibility of mild and malignant process differentiation, which in various reports ranged from $47 \%$ to $90 \%[1,6]$. The first ultrasound procedure in the presented case suggested the presence of alodgement, which was not seen in the following USG. The reason for this phenomenon might have been the fact that the deposits removed during the first ERCP may have been denser, and the density of concrements localised in the proximal part was similar to the surrounding bile. For that reason, they could not be visualised in either ultrasound examination.

The efficacy of computed tomography in biliary duct stone detection also depends on the composition. Hard concrements with significant contents of calcium, presenting about $20 \%$ of all types of deposits, are relatively easy to depict in the form of hyperdense formations
[3]. Soft, low-density deposits, built mainly from calcium bilirubinate or cholesterol, are more difficult to demonstrate. Twenty-seven perecnt of stones in this group present various densities of the core and the outer layer, $23 \%$ are homogenous, $15.4 \%$ are comparable to soft tissues and $34.6 \%$ are isodense in relation to the surrounding bile and, therefore, are invisible in examination without contrast agent [3, 7]. The possibility of concrement visualisation is also affected by the relative proportion of CBD and deposit diameter. This explains the so-called "target sign": the low-density halo of bile surrounding the deposit. This phenomenon is not visible in $30 \%$ of stones, which are wedged and can only be diagnosed on the basis of high-density foci visible in the expected course of CBD [3, 7]. Overall, the sensitivity and specificity of CT in the detection of biliary duct lithiasis is $24 \%, 6-88 \%$ and $84 \%$, respectively $[1,8]$. The disadvantage of classical CT is the lack of biliary duct anatomy visualisation. This fact, in the case of anomalies, may lead to interpretational mistakes. Consequently, the cholangiography $\mathrm{CT}$ technique improves detection of lithiasis in $85-95.5 \%$ of cases with a specificity of $96.6-100 \%$ [1]. However, CT is contraindicated in cases with increased bilirubin levels (exceeding 2.9$4.1 \mathrm{mg} / \mathrm{dl}$ ), liver parenchyma damage, renal failure with creatinine over $2 \mathrm{mg} / \mathrm{dl}$ and allergy to contrast agents (1\% of patients) [1]. The newest modification is virtual cholangiography CT. Nonetheless, the visualisation of the intrahepatic tree is correct only in $45-70 \%$ of cases [1]. The negative CT result in the described case can be explained by the low-density deposit and engagement of the whole CBD lumen, precluding "target sign" phenomenon. Cholangiography CT and its virtual modification was not possible because the contrast agents used in this examination are not available in Poland.

The following imaging technique is MRCP. The less invasive character of such a study gives it an advantage over CT or MRCP, because sedation, intravenous contrast or fluoroscopy exposure are avoided in this case $[1,2,4,9]$. In the MRCP study bile is high density, and the deposits are low density; this difference allows for $73-97 \%$ sensitivity and $87.5-96 \%$ specificity of the concrement detection [4]. However, the bigger the concrements, the lower the efficacy of the method, and the results for deposits over $10 \mathrm{~mm}$, between $6 \mathrm{~mm}$ and $10 \mathrm{~mm}$, and below $10 \mathrm{~mm}$ in size are 67-100\%, $89-94 \%$ and $33-71 \%$, respectively $[1,4]$. Another limitation, especially in older devices, is low image quality, preventing deposit dimensions and biliary tree contour assessment. The presence of air in the vicinity of the lumen of biliary duct, haemobilia, biliary flow disorders and vessel or diverticulum compression in the duodenum may also induce interpretation difficulties [1,9]. 
The false positive results of non-existent deposits might apply for $13 \%$ of the examined patients. The efficacy of the method dependents also on CBD diameter, because the lower the diameter, the more decreased the signal intensity $[2,3,8]$. Thus, MRCP credibility in mild and malignant changes causing stenosis differentiation is $58-88 \%[1,9]$. In the first procedure of the presented case, the deposits were found in the distal part of the $\mathrm{CBD}$, from where they were removed during previous ERCP. The reason of such an interpretation of the image might have been the presence of air in the distal part of the CBD, as a consequence of former sphincterotomy. The second study was also not able to differentiate between the deposits and the air, proving the deficiency of this technique in the described area and its limited credibility in patients after sphincterotomy or CBD prosthesis procedures.

The endoscopic ultrasonography (EUS) with $1 \mathrm{~mm}$ resolution seems to be the best available imaging technique, and it is especially useful in the diagnosis of small deposits [1]. It is less invasive than ECPR; however, it is limited by the operator's experience, necessity of sedation and partial access limited to $8-10 \mathrm{~cm}$. Additionally, the presence of air in biliary ducts, prosthesis and surgical clips or calcifications in the pancreas or duodenal diverticula pose further interpretational difficulties [1]. The sensitivity of stone detection by the method is $84-100 \%$ and specificity is $95-100 \%[1,6,8]$. The results are better than USG effects (sensitivity 63\%) or CT (sensitivity 71\%) and comparable to ECPR [8]. The USG and CT procedures are valid only in $50 \%$ of CBD deposits resembling tumours. In such cases, EUS is particularly useful. This method is highly sensitive (97\%) and specific (88\%) in extrahepatic biliary duct stenosis detection, although it is less efficient in the proximal part [1, 8-10]. The study, in comparison to ERCP, provides additional data in $75 \%$ of patients and allows a change in treatment method in $32 \%$ of cases [9]. This type of examination was not performed in the analysed case because of limited availability. However, it should be noted that the diagnostic problem in our case applied to the proximal part of the biliary tree, where the efficacy of the method is primarily lower.

Intraductal ultrasound (IDUS) is another imaging technique utilising higher frequencies $(12-30 \mathrm{MHz})$ in comparison to EUS, allowing for better resolution at the expense of lower penetration (1-3 cm outside the CBD lumen). It is recommended particularly in suspicion of choledocholithiasis affecting very small diameter ducts and Mirrizi syndrome [10]. Additionally, it allows for better differentiation of stenosis type, exclusively in the proximal part of biliary ducts. The sensitivity of the method in this region, in addition to ECPR, is $88-90 \%$ and is better than MRCP $[1,6]$. The basic disadvantages are its high cost, superficial penetration of the image and high operator-experience dependence [1]. It seems that the technique could have been useful in the analysed case; however, as far as we know the method is not performed in biliary duct diagnostics in Poland.

Contrast agent introduction to biliary ducts in the course of ERCP is possible in almost $100 \%$ of patients with biliary tree widening and in about $69-80 \%$ patients with normal diameter biliary ducts [1]. Nevertheless, the results rely on the extent of contrast filling, related to the amount of contrast agent, the position of the patient and the type of analgesia [1-3, 7]. The diagnostic difficulties occur in the differentiation of air or biliary stones, and the type of stenosis [1, 2, 11]. Mild changes visualised in cholangiography are usually multilevel with a smooth surface. Nonetheless, the early stages of hepatocellular carcinoma, biliary vesicle or pancreatic carcinoma growing in the vicinity may also induce slight compression of the duct lumen, inducing smooth stenosis. The pathomorphological structure of the tumour also affects cholangiography image. The most common biliary duct tumour, adenocarcinoma, occurring in $82.9 \%$ of patients, in half of the cases can assume the infiltrating form of a smooth stenosis, whereas the nodular form can imitate a deposit [2, 11]. Even so, the atypical concrements filling the whole lumen of the duct may mimic smooth stenosis in 1.3-3\% of patients with lithiasis [3].

The diagnostics should also consider the presence of thrombi, papilloma, air, improper contrast filling over the stenosis level, compression by the common hepatic artery (15-20\% of patients) and papilla of Vater spasm, resembling distal stones [3]. Consequently, the ECPR sensitivity of biliary lithiasis detection ranges from $78.9 \%$ to $91.7 \%$, and specificity is about $98 \%[1,10]$. However, only $5.2-30 \%$ of the patients remain questionable after this procedure [11].

In the first examination, typical deposits were removed from the distal part of the CBD. The statement of proximal stenosis without wrapping features suggested the coexistence of a different pathology. Therefore, the patient was subject to complementary diagnostics, although further USG, CT and double MRCP did not resolve these doubts. Moreover, none of the exams noted the presence of stenosis in the proximal part of the CBD, and one MRCP pointed to the widening of the intrahepatic tree. At the time of the third ECPR, diagnosis was performed; the stenosis was caused by a wrapped deposit, which was fragmented probably by the prosthesis implanted in the CBD.

Lie et al. reported similar sequences of events, showing that 6, 12 and 24 months after prosthetics 
$6 \%, 21 \%$ and $42 \%$, respectively, of the deposits undergo defragmentation, and the dimensions of the remaining material decreases by $60 \%$ [12]. Further data on the stenosis type can be achieved by direct cholangioscopic observation of biliary ducts. The method allows additionally for eye-guided biopsy sampling and newly described image analysis in intraductal confocal microscopy. Serious limitations of the procedure include its cost, manoeuvrability, difficulties in mother-child technique, limited optical resolution, high chance of damaging the cholangioscope, and the necessity for two experienced endoscopists to perform the procedure. The reported patient was qualified to such proceedings due to pertaining doubts, but the antecedent cholangiogram resolved the reason of the stenosis. Therefore, further diagnostics were abandoned.

The other method enabling obtainment of additional data may be cytologic or pathomorphological examination using the material samples obtained from the stenosis region by brush swabs, fine needle aspiration biopsy, biopsy, material achieved from the removed prosthesis, and brush swabs following introductory stenosis widening in order to reveal deeper tissues $[1,10]$. However, the sensitivity of single procedures ranges from $20 \%$ to $60 \%$, and the combination of two or more methods improves the efficacy of cholangiocarcinoma detection from $44 \%$ to $100 \%$. In addition, tissue sampling inside the biliary ducts guided only by fluoroscopy is connected with a risk of retroperitoneal perforation $[1,10]$. For this reason, in our case, material sampling in the region of the stenosis should have been cholangioscopy-guided [3].

\section{Conclusions}

Deposits that imitate common bile duct stenosis are a rare phenomenon, difficult to diagnose. This option should be taken into account during differential diagnostics of CBD stenosis.

\section{References}

1. Tse F, Barkun J, Romagnuolo J, et al. Nonoperative imaging techniques in suspected biliary tract obstruction HPB. (Oxford) 2006; 8: 409-25.

2. Bipat S, Phoa SS, van Delden OM, et al Ultrasonography, computed tomography and magnetic resonance imaging for diagnosis and determining resectability of pancreatic adenocarcinoma: a meta-analysis. J Comput Assist Tomogr 2005; 29: 438-45.

3. Baron R, Campell W. Nonneoplastic disease of the bile ducts. In: Margulis and Burhenne's Alimentary tract radiology. Freeny P, Stevenson G (eds.). Mosby 1989; 1294-343.

4. Griffin N, Wastle ML, Dunn WK, et al. Magnetic resonance cholangiopancreatography versus endoscopic retrograde cholan- giopancreatography in the diagnosis of choledocholithiasis. Eur J Gastroenterol Hepatol 2003; 15: 809-13.

5. Aslan F, Arabul M, Alper E, et al. The impact of periampullary diverticula on the endoscopic treatment of choledocholithiasis. Prz Gastroenterol 2012; 7: 281-5.

6. Tamada K, Tomiyama T, Wada S, et al. Endoscopic transpapillary bile duct biopsy with the combination of intraductal ultrasonography in the diagnosis of biliary strictures. Gut 2002; 50: 326-31.

7. Cabada GT, Sarria Octavio de Toledo L, Martinez-Berganza Asensio MT, et al. Helical CT cholangiography in the evaluation of the biliary tract: application to the diagnosis of choledocholithiasis. Abdom Imaging 2002; 27: 61-70.

8. Ainsworth AP, Rafaelsen SR, Wamberg PA, et al. Is there a difference in diagnostic accuracy and clinical impact between endoscopic ultrasonography and magnetic resonance cholangiopancreatography? Endoscopy 2003; 35: 1029-32.

9. Materne R, van Beers BE, Gigot JF, et al. Extrahepatic biliary obstruction: magnetic resonance imaging compared with endoscopic ultrasonography. Endoscopy 2000; 32: 3-9.

10. Farrell RJ, Jain AK, Brandwein SL, et al. The combination of stricture dilation, endoscopic needle aspiration, and biliary brushings significantly improves diagnostic yield from malignant bile duct strictures. Gastrointest Endosc 2001; 54: 587-94.

11. Pasanen P, Partanen K, Pikkarainen P, et al. Ultrasonography, CT, and ERCP in the diagnosis of choledochal stones. Acta Radiol 1992; 33: 53-6.

12. Li KW, Zhang XW, Ding J, et al. A prospective study of the efficacy of endoscopic stenting on common bile duct stones. J Dig Dis 2009; 10: 328-31.

Received: 26.09 .2011

Accepted: 27.03.2012 